\title{
A GENERAL METHOD OF DETECTING THE ADDITIVE, DOMINANCE AND EPISTATIC VARIATION THAT INBRED LINES CAN GENERATE USING A SINGLE TESTER
}

\author{
G. S. CHAHAL and J. L. JINKS \\ Department of Genetics, University of Birmingham, Birmingham B15 2TT
}

Received 9.v.77

\begin{abstract}
SUMmaRY
A new experimental design and analysis for investigating samples of inbred lines is described which avoids the difficulties that can arise in choosing adequate testers and the consequences of using inadequate testers while retaining most of the advantages of the triple test-cross design. The new design requires only a single inbred tester and providing that the sample of inbreds is in linkage equilibrium it can have any genotype. The design consists of producing the $F_{1}$ crosses between each inbred line in the sample and the tester, and then backcrossing each $F_{1}$ to its two inbred parents. Three sets of comparisons among the resulting parental, $F_{1}$ and backcross families provide tests for epistasis and in its absence equally reliable and direct tests and estimates of the additive $D$ and dominance $H$ components of variation.

If the sample of inbred lines is not in linkage equilibrium, the estimates of $D$ and $H$ may be differentially inflated or deflated, but this can be minimised if dominance is largely unidirectional by choosing a tester with an extreme phenotype. The design itself provides a test of the direction of the dominance.

The new analysis is illustrated by the reanalysis of portions of the $F_{1}, F_{2}$, $B_{1}$ and $B_{2}$ families of a diallel set of crosses between eight inbred lines of Nicotiana rustica grown in 1952 and 1953 which have the appropriate structure. The results show good agreement with those of previous analyses based upon more extensive data and suggest that the new analysis is a viable alternative to other test-cross designs.
\end{abstract}

\section{INTRODUCTION}

THE breeding of autogamous crop plants usually starts from a collection of inbred lines from different sources. These may be utilised directly for cultivation after screening, used to synthesise commercial hybrids or to create new genetic variability for the selection of superior inbreds. The first involves the direct exploitation of the additive genetic variance while the choice between the other two should depend on the relative magnitudes of the additive and dominance components of gene action. Indeed, if estimates of the additive, dominance and epistatic components of variability are available we can not only objectively assess the relative advantages and disadvantages of hybrids versus inbreds but we can also predict the probabilities of obtaining inbreds which are superior to the hybrids or to the original inbreds (Jinks and Perkins, 1972; Jinks and Pooni, 1976; Pooni and Jinks, 1977; Pooni, Jinks and Cornish, 1977).

The best method currently available for assessing these components of variation and in addition the magnitude and direction of genotype $\times$ environment interactions and linkage is the triple test-cross of Kearsey and Jinks (1968) and its extensions by Jinks and Perkins (1970) and Perkins and Jinks $(1970,1971)$. This approach has been simplified and modified in a 
number of ways for the restricted case of populations of inbred lines (Jinks, Perkins and Breese, 1969; Virk and Jinks, 1977; Jinks and Virk, 1977). However, for an arbitrary collection of inbred lines, choosing adequate testers, testing their adequacy and overcoming any biases introduced by their inadequacies can raise difficulties during the design, analysis and interpretation levels (Jinks and Virk, 1977). Furthermore, should the analyses reveal inadequacies in the testers many of the advantages of this design relative to others are reduced. These advantages can always be regained, of course, by adopting a more elaborate design extending over a number of generations, for example, a partial or complete diallel set of crosses continued to the $F_{2}$ and first backcrosses, $B_{1}$ and $B_{2}$, of each of the initial crosses (Jinks, 1956; Jinks and Stevens, 1959). But in practice what is required is a design that retains as far as possible the simplicity of the triple test-cross consistent with providing direct tests of the significance of all of the components and independent estimates of each component with comparable reliability. Such a compromise has been found from within the more complex analysis of Jinks and Stevens (1959).

\section{EXPERIMENTAL DESIGN}

Each line $\left(P_{i}\right)$ of a sample $n$ of a collection of inbred lines is crossed to one arbitrarily chosen inbred line $\left(P_{c}\right)$ to produce an array of $n F_{1 i}$ families. Each $F_{1 i}$ family is then backcrossed to each of its parents one of which is unique to that family $\left(P_{i}\right)$ and one of which is common to all families $\left(P_{c}\right)$ to produce two first backcross families $B_{1 i}\left(F_{1 i} \times P_{i}\right)$ and $B_{c i}\left(F_{1 i} \times P_{c}\right)$, respectively. The experiment then consists of the $P_{c}$ family and $n$ of each of the $P_{i}, F_{1 i}, B_{2 i}$ and $B_{c i}$ families giving a total of $4 n+1$ families, each of which is replicated by raising either $r$ plots or preferably $r$ individuals in a randomised block design. Alternatively we can include $n r$ replicates of $P_{c}, r$ of which are allocated at random to each of the $n$ sets of other families for making the various comparisons (see Section 3).

\section{MODEL AND ANALYSIS}

The analysis may be divided into two parts, the first concerned with the test for epistasis, the second with testing for and estimating the additive and dominance components of variation should epistasis prove to be absent.

\section{(i) Test for epistasis}

The test for epistasis is based on the standard backcross scaling tests (Mather and Jinks, 1971). In table 1 are given the expectations for these scaling tests for the two backcrosses, $B_{1 i}$ and $B_{c i}$, for each of the four possible genotypes for $P_{i}$ in respect of two loci, and for the the corresponding four possible genotypes for the tester, $P_{c}$. In the absence of epistasis these tests have an expectation of zero irrespective of the genotype of the inbred line, $P_{i}$ or of the tester, $P_{c}$. This will be true irrespective of the number of pairs of loci since all possibilities in respect of any pair of loci are represented in table 1 .

We could proceed by carrying out the $2 n$ individual scaling tests or preferably the $n$ joint scaling tests combining $B_{1 i}$ and $B_{c i}$ for the $i$ th set for 
TABLE 1

The contribution of additive, dominance and digenic interaction parameters to the comparisons, (i) $2 \overline{\mathrm{B}}_{11}-\overline{\mathrm{F}}_{1 \mathrm{i}}-\overline{\mathrm{P}}_{\mathbf{1}}$ and (ii) $2 \overline{\mathrm{B}}_{\mathrm{e1}}-\overline{\mathrm{F}}_{1 \mathrm{1}}-\overline{\mathrm{P}}_{\mathrm{c}}$ for all possible $\mathrm{P}_{\mathbf{c}}$ genotypes in respect of two pairs of genes

\begin{tabular}{|c|c|c|c|c|c|c|}
\hline \multicolumn{3}{|c|}{$\underbrace{P_{t}}$} & \multicolumn{4}{|c|}{ Possible $P_{c}$ genotypes } \\
\hline Frequency* & Genotype & Comparison & $A A B B$ & $A A b b$ & $a a B B$ & $a a b b$ \\
\hline$\alpha$ & $A A B B$ & (i) & $\begin{array}{l}\mathbf{0} \\
\mathbf{0}\end{array}$ & $\begin{array}{l}\mathbf{0} \\
\mathbf{0}\end{array}$ & $\begin{array}{l}0 \\
0\end{array}$ & $\begin{array}{l}\frac{1}{2}\left(-i_{a b}+j_{a b}+j_{b a}-l_{a b}\right) \\
\frac{1}{2}\left(-i_{a b}-j_{a b}-j_{b a}-l_{a b}\right)\end{array}$ \\
\hline$\beta$ & $A A b b$ & (i) & $\begin{array}{l}0 \\
0\end{array}$ & $\begin{array}{l}\mathbf{0} \\
0\end{array}$ & $\begin{array}{l}\frac{1}{2}\left(i_{a b}+j_{a b}-j_{b a}-l_{a b}\right) \\
\frac{1}{2}\left(i_{a b}-j_{a b}+j_{b a}-l_{a b}\right)\end{array}$ & $\begin{array}{l}0 \\
0\end{array}$ \\
\hline$\gamma$ & $a a B B$ & (i) & $\begin{array}{l}0 \\
0\end{array}$ & $\begin{array}{l}\frac{1}{2}\left(i_{a b}-j_{a b}+j_{b a}-l_{a b}\right) \\
\frac{1}{2}\left(i_{a b}+j_{a b}-j_{b a}-l_{a b}\right)\end{array}$ & $\begin{array}{l}0 \\
0\end{array}$ & $\begin{array}{l}0 \\
0\end{array}$ \\
\hline$\delta$ & $a a b b$ & (i) & $\begin{array}{l}\frac{1}{2}\left(-i_{a b}-j_{a b}-j_{b a}-l_{a b}\right) \\
\frac{1}{2}\left(-i_{a b}+j_{a b}+j_{b a}-l_{a b}\right)\end{array}$ & $\begin{array}{l}0 \\
0\end{array}$ & $\begin{array}{l}0 \\
0\end{array}$ & $\begin{array}{l}0 \\
0\end{array}$ \\
\hline
\end{tabular}

$i=1$ to $n$ (Mather and Jinks, 1971). The former would lead to $2 n$ individual $t$ tests the latter to $n$ individual $\chi^{2}$ tests each for 2 degrees of freedom. A test which combined all these individual tests of significance is clearly preferable and this can be achieved within the context of an analysis of variance.

Taking first the $n$ sets of comparisons of the kind

$$
2 \bar{B}_{1 i}-F_{1 i}-\bar{P}_{i}=A_{i}
$$

from the sum of $A_{i}^{2}$ over the $n$ sets $\left(\sum_{i=1}^{n} A_{i}^{2}\right)$ we can obtain a mean square for $n$ degrees of freedom. This can be tested against the mean square derived from pooling the corresponding variances of $A_{i}$ which are obtained in the usual way from the variances of the $B_{1 i}, F_{1 i}$ and $P_{i}$ family means (Mather and Jinks, 1971).

For the $n$ sets of comparisons of the kind

$$
2 \bar{B}_{c i}-\bar{F}_{1 i}-\bar{P}_{c}=B_{i}
$$

$\bar{P}_{c}$ appears in every set and hence unless we raise $n$ independently randomised sets of $r$ replicates of $P_{c}$, each allocated to a particular one of the $n$ sets (Section 2), the $n B_{i}$ values will not be independent. Given these $n$ sets of $P_{c}$, the analysis of the $B_{i}$ values proceeds exactly as for the corresponding $A_{i}$ 's. Alternatively, however, if we have only one estimate of $\bar{P}_{c}$ we can rewrite this comparison as

$$
2 \bar{B}_{c i}-\bar{F}_{1 i}=B_{i}+\bar{P}_{c}=B_{i}+\text { constant }
$$

Our test is then based on the mean square derived from the sum of squares of the deviations of the $n$ values of $\left(2 \bar{B}_{c i}-\bar{F}_{1 i}\right)$ around their own mean for $n-1$ degrees of freedom. This can be tested against the mean square derived in the usual way from the variances of the $B_{c i}$ and $F_{1 i}$ family means.

\section{(ii) Additive and dominance components}

If epistasis is absent two further orthogonal comparisons provide unique tests for the additive and dominance components of variation. These are summarised in table 2 for all possible genotypes in respect of two loci for $P_{i}$ and for the corresponding possible genotypes for $P_{c}$. For each comparison we can compute a sum of squares of deviations of the $n$ values from their own 
TABLE 2

The contribution of additive and dominance parameters to the comparisons $(i) \overline{\mathrm{B}}_{\mathrm{c} 1}-\overline{\mathrm{B}}_{1 \mathrm{i}}-\overline{\mathrm{P}}_{\mathrm{c}}+\overline{\mathrm{P}}_{\mathrm{i}}$ and $(i i)$ $\overline{\mathrm{B}}_{\mathrm{ci}}+\overline{\mathrm{B}}_{1 \mathrm{i}}-\overline{\mathrm{P}}_{\mathrm{c}}-\overline{\mathrm{P}}_{\mathbf{i}}$, for all possible $\mathbf{P}_{\mathrm{c}}$ genotypes in respect of two pairs of genes

Possible $P_{c}$ genotypes

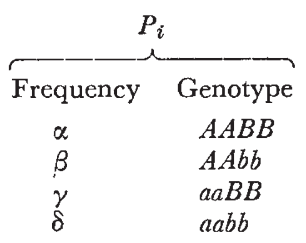

\begin{tabular}{|c|c|c|c|c|c|c|c|}
\hline \multicolumn{2}{|c|}{$\underbrace{A A B B}$} & \multicolumn{2}{|c|}{$\underbrace{A A b b}$} & \multicolumn{2}{|c|}{$\underbrace{a a B B}$} & \multicolumn{2}{|c|}{$\underbrace{a a b b}$} \\
\hline (i) & (ii) & (i) & (ii) & (i) & (ii) & (i) & (ii) \\
\hline $\begin{array}{c}0 \\
-d_{b} \\
-d_{a} \\
-d_{a}-d_{b}\end{array}$ & $\begin{array}{c}0 \\
h_{b} \\
h_{a} \\
h_{a}+h_{b}\end{array}$ & $\begin{array}{c}d_{b} \\
0 \\
-d_{a}+d_{b} \\
-d_{a}\end{array}$ & $\begin{array}{c}h_{b} \\
0 \\
h_{a}+h_{b} \\
h_{a}\end{array}$ & $\begin{array}{c}d_{a} \\
d_{a}-d_{b} \\
0 \\
-d_{b}\end{array}$ & $\begin{array}{c}h_{a} \\
h_{a}+h_{b} \\
0 \\
h_{b}\end{array}$ & $\begin{array}{c}d_{a}+d_{b} \\
d_{a} \\
d_{b} \\
0\end{array}$ & $\begin{array}{c}h_{a}+h_{b} \\
h_{a} \\
h_{b} \\
0\end{array}$ \\
\hline
\end{tabular}

mean for $n-1$ degrees of freedom. We can also compute a corresponding sum of squares for the replicate error whose exact form and degrees of freedom will depend on the experimental design. The expected mean squares in all cases would reduce to the following form, where $r$ is the number of replications.

\section{Comparison}

1. $\bar{B}_{c i}-\bar{B}_{1 i}-\bar{P}_{c}+\bar{P}_{i}$ (Additive)

2. $\bar{B}_{c i}+\bar{B}_{1 i}-\bar{P}_{c}-\bar{P}_{i}$ (Dominance)

Error
Expected mean square

$$
\begin{aligned}
& \sigma^{2}+4 r \sigma_{1}^{2} \\
& \sigma^{2}+4 r \sigma_{2}^{2} \\
& \sigma^{2}
\end{aligned}
$$

Hence we can test the significance of, and estimate $\sigma_{1}^{2}$ and $\sigma_{2}^{2}$ independently and with equal precision. Furthermore, reference to tables 2 and 3 shows that in all circumstances $\sigma_{1}^{2}$ reflects only the additive genetic effects $(d)$ while $\sigma_{2}^{2}$ reflects only the dominance effects $(h)$. Where we have only a single estimate of $P_{c}$ for these comparisons, this, being a constant in all the

\section{TABLE 3}

Estimates of the additive and dominance components of variation and tests of epistasis for final height in 1952 and 1953

\begin{tabular}{|c|c|c|c|c|}
\hline \multirow{2}{*}{$\begin{array}{l}\text { Complete } \\
F_{1} \text { diallel }\end{array}$} & \multirow{2}{*}{$\begin{array}{c}\text { Complete } F_{1} \text {, } \\
F_{2}, B_{1} \text { and } \\
B_{2} \text { diallel }\end{array}$} & \multirow{2}{*}{$\begin{array}{l}F_{1}, F_{2}, B_{1} \\
\text { and } B_{2} \text { of } \\
V 38 \text { array }\end{array}$} & \multicolumn{2}{|c|}{ New analysis } \\
\hline & & & $P_{c}=V 38$ & $P_{c}=V 12$ \\
\hline ignificant* & Significant & Non-significant & Non-significant & Significant \\
\hline $43 \cdot 00$ & $37 \cdot 13 \pm 3 \cdot 72$ & $44 \cdot 59 \pm 3 \cdot 20$ & $46 \cdot 62$ & \\
\hline $108 \cdot 99$ & $76 \cdot 74 \pm 20 \cdot 68$ & $38 \cdot 15 \pm 20 \cdot 19$ & $37 \cdot 72$ & $132 \cdot 35$ \\
\hline $\mathrm{I} \cdot 59$ & $1 \cdot 44$ & 0.92 & 0.90 & $\mathrm{~J} \cdot 05$ \\
\hline
\end{tabular}
from previous analyses and from the new analysis

Previous analyses (Jinks 1954, 1956)

1953

Year

Component

Epistasis

$D$
$\frac{H}{\sqrt[H]{ } / D}$

$$
\begin{gathered}
\text { Epistasis } \\
D \\
\sqrt{ } \frac{H}{H / D}
\end{gathered}
$$

Significant*
$64 \cdot 66$
$23 \mathrm{I} \cdot 04$
$\mathrm{I} \cdot 94$

\section{Significant}

$55 \cdot 25 \pm 5 \cdot 07$

$222 \cdot 98 \pm 28 \cdot 16$

$2 \cdot 0 \mathrm{I}$
Non-significant Non-significant

$6 \mathrm{I} \cdot 20 \pm 5 \cdot 12$

$94 \cdot 41 \pm 32 \cdot 28$

$\mathrm{I} \cdot 24$
$57 \cdot 75$

$8 \mathrm{I} \cdot 26$

$\mathrm{I} \cdot \mathrm{I} 9$

* These significances could result from epistasis, linkage disequilibrium or both but in all other analyses they test specifically epistasis only. 
comparisons, can be omitted and the expectations amended accordingly, the coefficients of $\sigma_{1}^{2}$ and $\sigma_{2}^{2}$ both becoming $3 r$.

It is clear from table 2 that while $\sigma_{1}^{2}$ and $\sigma_{2}^{2}$ depend solely on the $d$ and $h$ effects of the genes, respectively, their expectations will depend on the relative frequencies with which the different $P_{i}$ genotypes are included in the sample of inbreds and in some circumstances on the genotype of the tester, $P_{c}$ also. For example, if we assume that the sample is in linkage equilibrium $\left(\alpha=u_{a} u_{b}, \beta=u_{a} v_{b}, \gamma=v_{a} u_{b}\right.$ and $\left.\delta=v_{a} v_{b}\right)$ but with arbitrary gene frequencies of $u_{a}$ for $A, v_{a}$ for $a$ and $u_{b}$ for $B$ and $v_{b}$ for $b$, then for a single pair of loci the expectations are

$$
\begin{aligned}
& \sigma_{1}^{2}=f u_{a} v_{a} d_{a}^{2}+f u_{b} v_{b} d_{b}^{2} \\
& \sigma_{2}^{2}=f u_{a} v_{a} h_{a}^{2}+f u_{b} v_{b} h_{b}^{2},
\end{aligned}
$$

where $f=\frac{1}{16}$ if $\bar{P}_{c}$ is included and $\frac{1}{8}$ if $\bar{P}_{c}$ is excluded from the comparisons irrespective of the genotype of $P_{c}$ itself.

For many such pairs of loci

$$
\begin{aligned}
& \sigma_{1}^{2}=f \Sigma u_{i} v_{i} d_{i}^{2}=f \frac{1}{4} D \\
& \sigma_{2}^{2}=f \Sigma u_{i} v_{i} h_{i}^{2}=f \frac{1}{4} H
\end{aligned}
$$

for all testers.

If we now relax the assumption of linkage equilibrium so that $\alpha \neq u_{a} u_{b}$, $\beta \neq u_{a} v_{b}, \gamma \neq v_{a} u_{b}$ and $\delta \neq v_{a} v_{b}$ the expectations of $\sigma_{1}^{2}$ and $\sigma_{2}^{2}$ will depend on the genotype of the tester $P_{c}$. For example, if the tester is $A A B B$

$$
\sigma_{1}^{2}=f u_{a} v_{a} d_{a}^{2}+f u_{b} v_{b} d_{b}^{2}+2 f\left(\delta-v_{a} v_{b}\right) d_{a} d_{b}
$$

and

$$
\sigma_{2}^{2}=f u_{a} v_{a} h_{a}^{2}+f u_{b} v_{b} h_{b}^{2}+2 f\left(\delta-v_{a} v_{b}\right) h_{a} h_{b}
$$

whereas if the tester is $A A b b$

$$
\begin{aligned}
& \sigma_{1}^{2}=f u_{a} v_{a} d_{a}^{2}+f u_{b} v_{b} d_{b}^{2}-2 f\left(\gamma-v_{a} u_{b}\right) d a d b \\
& \sigma_{2}^{2}=f u_{a} v_{a} h_{a}^{2}+f u_{b} v_{b} h_{b}^{2}+2 f\left(\gamma-v_{a} u_{b}\right) h a h b
\end{aligned}
$$

The expectation for the other testers, $a a b b$ and $a a B B$ are obtained by substituting $\left(\alpha-u_{a} u_{b}\right)$ for $\left(\delta-v_{a} v_{b}\right)$ and $\left(\beta-u_{a} v_{b}\right)$ for $\left(\gamma-v_{a} u_{b}\right)$ in these expectations, respectively. Since at equilibrium $\delta=v_{a} v_{b}, \quad \gamma=v_{a} u_{b}$, $\alpha=u_{a} u_{b}$ and $\beta=u_{a} v_{b}$ these additional terms are simple functions of the linkage disequilibrium. In the presence of linkage disequilibrium, however, the form of the expectations differs according to whether the single tester $P_{c}$ has the pairs of genes in association $(A A B B$ or $a a b b)$ or in dispersion $(A A b b$ or $a a B B)$. Furthermore, the coefficients of the $d$ terms in $\sigma_{1}^{2}$ and of the $h$ terms in $\sigma_{2}^{2}$ are no longer necessarily identical. All testers therefore, will do equally well when the $P_{i}$ sample is in equilibrium. When this is not so a tester with the maximum number of gene pairs in association and hence with an extreme phenotype is preferable because the most critical decisions usually arise when there is strong directional dominance leading to heterosis (see Introduction). We can in fact obtain a good indication as to whether the dominance is mainly unidirectional or ambidirectional from the signs 
of the deviations obtained from the dominance comparison (table 2). If all $n$ values for this comparison have the same sign there is clearly a strong unidirectional component. If on the other hand some are positive and some negative the unidirectional element must be weak or even absent if the positive and negative deviations exactly balance one another.

\section{Alternative sets of Comparisons}

In Section 3 we presented one set of comparisons among the $P_{c}, P_{i}$, $F_{1 i}, B_{c i}$ and $B_{1 i}$ families which satisfies all our requirements. In all, however, we have examined 63 different comparisons, if we count different signs and coefficients, among these families and have found only one further orthogonal pair which can provide independent tests of significance and estimates of $D$ and $H$; these are $\bar{B}_{c i}+\bar{B}_{1 i}-\bar{P}_{i}-F_{1 i}$ and $\bar{B}_{c i}-\bar{B}_{1 i}+\bar{P}_{i}-\bar{F}_{1 i}$, respectively. In terms of the simple theory we have developed there is no reason to prefer either one set or the other for estimating $D$ and $H$. However, because of the symmetry of the earlier set of comparisons it will in general be more robust in the presence of complications. For example, with the earlier set of comparisons it is sufficient in the presence of maternal effects to use $P_{i}$ as the maternal parent of $B_{1 i}$ and $P_{c}$ as the maternal parent of $B_{c i}$ to eliminate these effects from the estimates of $D$ and $H$. Furthermore, with these comparisons the biases in the estimates of $D$ and $H$ in the presence of interactions are the familiar ones whose properties are well understood (see Mather and Jinks, 1971, Chapter 7).

\section{EXAMPLE}

For illustration we shall reanalyse data for final height from a diallel set of crosses between eight inbred lines of Nicotiana rustica carried out by Jinks between 1950 and 1953 . The parents and $F_{1}$ 's of this diallel set were grown in 1951 and again in 1952 and 1953 along with their $F_{2}$ and first backcross $\left(B_{1}\right.$ and $\left.B_{2}\right)$ families (see Jinks, 1954 and 1956, for details). The data for final height and for a number of other characters have been analysed as $F_{1}$ diallels (Jinks, 1954), as combined parental, $F_{1}, F_{2}, B_{1}$ and $B_{2}$ families of a diallel set (Jinks, 1956) and by a set of comparisons which separate additive, dominance and epistatic components of variation (Jinks and Stevens, 1959). The results of some of these earlier analyses are summarised in the first three columns of table 4. The first is the analysis of the complete $F_{1}$ diallel for 1952 and 1953 . This provides perfect fit estimates of $D$ and $H$ (as well as of other components which are not relevant) and tests of the adequacy of the additive, dominance model which in this context is a joint test of the independence of the genes in action (no epistasis) and in distribution (no linkage disequilibrium). The second is the analysis of the combined parental, $F_{1}, F_{2}, B_{1}$ and $B_{2}$ families for 1952 and 1953. This provides least squares estimates of $D$ and $H$ and of other components, and specific tests for epistasis as well as for other causes of inadequacy of the simple model. The third is a joint analysis of all the families belonging to one array of the diallel set, that with common parent $V 38$, the only one within which the additive, dominance model is adequate, using the single array analysis of Jinks (1955). This array is the obvious first choice for illustrating our new analysis. 
TABLE 4

The mean final height in inches and the number of plots of five plants on which the mean is based for the tester line, $\mathrm{P}_{c}$, the seven $\mathrm{P}_{1}$ lines and their $\mathrm{F}_{11}, \mathrm{~B}_{c \mathrm{1}}$ and $\mathrm{B}_{1 \mathrm{i}}$ families grown in 1952

$\begin{array}{rlcccc}P_{c} & V 38 & 35.90(2) & F_{1 i}\left(P_{i} \times P_{c}\right) & B_{c i}\left(F_{1 i} \times P_{c}\right) & B_{1 i}\left(F_{1 i} \times P_{i}\right) \\ P_{i} i=1 & V 2 & 33.50(2) & 34 \cdot 55(4) & 32 \cdot 55(4) & 33 \cdot 70(4) \\ 2 & V 5 & 38 \cdot 40(2) & 40 \cdot 80(4) & 36 \cdot 05(4) & 41 \cdot 90(4) \\ 3 & V 7 & 27 \cdot 40(2) & 35 \cdot 40(4) & 34 \cdot 10(4) & 32 \cdot 90(4) \\ 4 & V 12 & 51 \cdot 30(2) & 50 \cdot 60(4) & 40 \cdot 95(4) & 50 \cdot 35(4) \\ 5 & V 14 & 35 \cdot 80(2) & 36 \cdot 35(4) & 34 \cdot 70(4) & 40 \cdot 35(4) \\ 6 & V 29 & 39 \cdot 50(2) & 35 \cdot 30(4) & 32 \cdot 80(4) & 36 \cdot 45(4) \\ 7 & V 41 & 33 \cdot 30(2) & 33 \cdot 70(2) & 33 \cdot 65(4) & 33 \cdot 70(4)\end{array}$

If we now equate the relevant families of this array to those of the new design; V38 is the tester line, $P_{c}$, and the remaining seven inbred lines, provide a sample of $n=7$ of the inbred lines, $P_{i}$. There are then seven $F_{1 i}$ families $\left(P_{i} \times P_{c}\right.$ for $i=1$ to 7$)$. In the complete diallel these $F_{1}$ crosses were made reciprocally and we shall regard these as replicates in our subsequent analyses. Both reciprocals of each $F_{1 i}$ were themselves backcrossed as female parents to $P_{c}$ and to the corresponding $P_{i}$ to produce reciprocal (replicate) pairs of $B_{c i}$ and $B_{1 i}$ families. All $P_{c}, P_{i}, F_{1 i}, B_{c i}$ and $B_{1 i}$ families were grown simultaneously in 1952 and in 1953 in a randomised block design with two blocks, the unit of randomisation being a plot of five plants of the same family. The total number of plots of each family grown in 1952 are given in table 4 along with the mean final height of each family in inches. For reasons which have been discussed elsewhere we shall use a conservative estimate of the replicate error based upon the differences between reciprocal crosses rather than on the differences between blocks when both are available (Jinks, 1954, 1956).

Since the $W r, V r$ analysis and tests of goodness of fit of models to first and second degree statistics (Jinks, 1954, 1956) revealed no evidence of epistasis in any cross involving $P_{c}(V 38)$ it is not surprising that our test for epistasis based on an analysis of variance (Section 3 (i) ) was not significant $(\mathrm{P}>0.25)$. We can proceed therefore with the fitting of the additive, dominance model.

Using the comparisons described in Section 3 the expression appropriate for testing and estimating the additive and dominance effects in these data are those which omit $\vec{P}_{c}$ and these and their corresponding mean squares are given in table 5 . The 35 degrees of freedom for the error mean square

TABle 5

Analysis of variance for the additive and dominance comparisons for final height in 1952

$\begin{array}{lrccc}\quad \text { Item } & \text { d.f. } & \text { M.S. } & \text { P } & \text { EMS } \\ \text { 1. Additive }\left(\bar{B}_{c i}-\bar{B}_{1 i}+\bar{P}_{i}\right) & 6 & 12 \cdot 6447 & 0 \cdot 05 & \sigma^{2}+3 r \sigma_{1}^{2} \\ \text { 2. Dominance }\left(\bar{B}_{c i}+\bar{B}_{1 i}-\bar{P}_{i}\right) & 6 & 11 \cdot 1613 & 0 \cdot 05-0 \cdot 10 & \sigma^{2}+3 r \sigma^{2} \\ \text { Error } & 35 & 4.8751 & & \sigma^{2}\end{array}$

$$
\begin{array}{rlrl}
\hat{\sigma}_{1}^{2} & =1 \cdot 2949=\frac{1}{36} D & \hat{D}=46 \cdot 62 \\
\hat{\sigma}_{2}^{2}=1 \cdot 0477 & =\frac{1}{36} H \quad \hat{H}=37 \cdot 72 .
\end{array}
$$


are made up of 28 for the reciprocal differences within blocks of the seven $B_{c i}$ and seven $B_{1 i}$ families (two for each family) plus 7 degrees of freedom for block difference for the seven $P_{i}$ inbreds. While the dominance component is not formally significant $(P \gtrless 0.05)$ this merely reflects the inefficiency of the experimental designs used at that time so we shall proceed to estimate both of the genetical components. The estimates of $D$ and $H$ (tables 3 and 5) correspond very closely to those obtained from the previous analysis of the $V 38$ array. The corresponding 1953 data have also been reanalysed using the new method with the results shown in table 3 . They too correspond very closely with the results of previous analyses. Furthermore, in these data the dominance component was significant at the level of $\mathrm{P}=0 \cdot 05-0 \cdot 01$.

We can of course use the other arrays from the $8 \times 8$ diallel set of crosses to illustrate our new analysis but in so far as previous analyses have shown that they include epistatic crosses our analyses should detect epistasis, and if we then proceed to estimate $D$ and $H$ they will be biased by this epistasis. To illustrate this we have analysed the 1952 data for the array of which $V 12$ is the common parent. The $W r, V r$ analysis and the joint scaling tests (Jinks, 1954, 1956) have shown that the crosses in this array have a high incidence of epistasis and our analysis of variance for epistasis confirms this $(P=0.05)$. Nevertheless, we have estimated the additive and dominance components both of which are significant $(\mathbf{P}<0.05)$ and their values are given in table 3 . As we expected they are both greatly inflated relative to the corresponding estimates for the $V 38$ array.

\section{Conclusions}

In this paper we have shown that for a population of inbred lines the problems created by inadequate testers can be completely avoided. At the cost of an additional generation of breeding, little or no increase is required in the size of the experiment or in the complexity of the breeding programme. While we have yet to test the new design and analysis on material specifically and optimally designed for the purpose, the results of reanalysing existing data which have an appropriate structure are encouraging and reproduce the conclusions drawn from more extensive data analysis. For the special case of populations of inbred lines, the new design and analysis is a viable alternative to the triple test-cross and its various modifications.

Acknowledgment.-This work was carried out while Dr G. S. Chahal was a Leverhulme Commonwealth/American Visiting Fellow.

\section{ReFERENCES}

JINKs, J. L. 1954. The analysis of continuous variation in a diallel cross of Nicotiana rustica varieties. Genetics, 39, 767-788.

JINKS, J. L. 1955. A survey of the genetical basis of heterosis in a variety of diallel crosses. Heredity, 9, 223-238.

Jinks, J. L. 1956. The $\mathrm{F}_{2}$ and backcross generations from a set of diallel crosses. Heredity, 10, 1-30.

JINKS, J. L., AND PERKINS, JEAN M. 1970. A general method for the detection of additive, dominance and epistatic components of variation. III. $\mathrm{F}_{2}$ and backcross populations. Heredity, 25, 419-429.

Jinks, J. L., AND PERKins, JEAN m. 1972. Predicting the range of inbred lines. Heredity, 28, 399-403. 
JiNks, J. L., PERkins, JeAn M., AND BReEse, E. L. 1969. A general method of detecting additive, dominance and epistatic variation for metrical traits. II. Application to inbred lines. Heredity, 24, 115-127.

JINKs, J. L., AND POONI, H. s. 1976. Predicting the properties of recombinant inbred lines derived by single seed descent. Heredity, 36, 253-266.

Jinks, J. L., AND steVENs, J. M. 1959. The components of variation among family means in diallel crosses. Genetics, 44, 297-308.

JINKs, J. L., AND VIRK, D. s. 1977. A modified triple test-cross analysis to test and allow for inadequate testers. Heredity, 39, 165-170.

KEARSEY, M. J., AND JINKs, J. L. 1968. A general method of detecting additive, dominance and epistatic variation for metrical traits. I. Theory. Heredity, 23, 403-409.

MATHER, K., AND JINKs, J. L. 1971. Biometrical Genetics. Chapman and Hall, London.

PERKINS, JEAN M., AND JINKS, J. L. 1970. Detection and estimation of genotype-environmental linkage and epistatic components of variation for a metrical trait. Heredity, 25, 157-177.

PERKINS, JEAN M., AND JINKS, J. L. 1971. Analysis of genotype-environment interaction in triple test cross data. Heredity, 26, 203-209.

POONI, H. s., AND JINks, J. L. 1977. Predicting the properties of recombinant inbred lines derived by single seed descent for two or more characters simultaneously. Heredity (in press).

POONI, H. S., JINKS, J. L., AND CORNISH, M. A. 1977. The causes and consequences of nonnormality in predicting the properties of recombinant inbred lines. Heredity, 38, 329-338.

VIRK, D. s., AND JINKs, J. L. 1977. The consequences of using inadequate testers in the simplified triple test-cross. Heredity, 38, 237-257. 